\title{
NR4A2 Expression Patterns in Mouse Models of Rheumatoid Arthritis
}

\author{
Jordan Everett ${ }^{\mathrm{a}}$, Ellen Gravallese ${ }^{\mathrm{b}}$ and Kimberlee S. Mix ${ }^{\mathrm{a}}$
}

Arthritis is a group of over 100 musculoskeletal disorders affecting approximately 50 million adults in the US. In an effort to develop new drugs to treat arthritis, we are exploring the function of the orphan nuclear receptor 4A2 (NR4A2), a transcription factor over-expressed in inflamed joints. The transcriptional targets of NR4A2 include angiogenesis factors and matrix metalloproteinases (MMPs). NR4A2 appears to have a deleterious effect in synoviocytes by promoting tissue degradation, while in chondrocytes it seems to have a protective function. Previous work on human synoviocytes has shown NR4A2 to rise early in response to inflammation, leading us to hypothesize that NR4A2 may be a preliminary mediator of arthritis. To test this hypothesis in vivo, we studied NR4A2 expression patterns in two mouse models of RA: Antigen Induced Arthritis (AIA) and Serum Transfer Arthritis (STA). Tissue sections were obtained from healthy and arthritic mice at early, mid, and late time-points following induction. Joint cross-sections were examined via immunohistochemical staining, and NR4A2 positive cells were quantified in synovial and cartilage tissues. In the AIA model, NR4A2 protein levels peaked in synovium at day 10 of disease (mid stage, $50 \%$ positive) and declined later in disease. In cartilage, protein levels reached a maximum at day 8 (early stage, $70 \%$ ) and subsequently declined as well. In contrast, NR4A2 was not expressed in the STA model, despite apparent joint degradation.

Keywords: NR4A2; immunohistochemistry; rheumatoid arthritis; transcription factor; synoviocyte; chondrocyte

\section{Introduction}

Arthritis is one of the most prevalent diseases in the United States. Approximately 1 in every 5 adults ( 50 million adults) and over 300,000 children in America are afflicted with some form of arthritis which broadly means a variety of musculoskeletal conditions that destroy bone, cartilage, muscle and other joint tissues (The Arthritis Foundation, 2011). Rheumatoid arthritis (RA) is an autoimmune disorder in which the body attacks its own joints with contributing factors from both one's environment and genetics (Asquith, Miller, McInnes, \& Liew, 2009). It is also the second most prevalent type of arthritis (affecting approximately 1.5 million in the United States alone); the most prevalent form is osteoarthritis (OA) (The Arthritis Foundation, 2011). OA is primarily caused by long term wear and tear of the joint, while RA has a more significant genetic component (Carr, 2003; Spector \& MacGregor, 2004)

Rheumatoid arthritis usually affects the small joints of the hands and feet, and it is much more prevalent in women than men. Within the joint, chronic inflammation causes swelling, cartilage degradation, bone erosion and severe pain (Mayo Clinic, 2013). This inflammation is mediated by a variety of cells responsible for the immune response and the cytokines and chemokines they produce. The exact cause of this aberrant immune activity is still unknown.

The immune cells that respond to the 'auto-antigen' (the antigen presented to immune cells as foreign but is not) perpetuate the inflammatory response and create a cycle of chronic destruction characteristic of RA (Frisenda, Perricone, \& Valesini, 2013). The most common immune cells present in a joint afflicted with RA cells are macrophages and monocytes, but mast cells, basophils, and eosinophils also contribute to the immune response. These cells secrete several inflammatory cytokines including tumor necrosis factor alpha
(TNF- $\alpha$ ) Interleukin-1 (IL-1), IL-6, and IL-8 (Harney, Newton, \& Wordsworth, 2003). These cytokines are expressed at low levels in healthy individuals, but levels increase significantly in RA joints. The induction pathway produces an exponential increase: TNF- $\alpha$ and the ILs expressed are inflammatory mediators which are produced by immune cells in response to auto-antigens, but the cells also respond to those same cytokines in a positive feedback loop to continuously increase the inflammatory response (Harney et al., 2003). In addition to the immune cells, the resident cells of the joint, the synoviocytes and chondrocytes, also exhibit altered activity in response to cytokine production. Synoviocytes grow rapidly and proliferate into the joint space; they also release more cytokines, particularly IL-1. IL-1 activates chondrocyte synthesis of Prostaglandin $\mathrm{E}_{2}\left(\mathrm{PGE}_{2}\right)$ and several neutral metalloproteinases; this causes degradation of the extracellular matrix of the cartilage due to the metalloproteinases and more inflammation via $\mathrm{PGE}_{2}$ a potent inflammatory mediator (Hulkower, Georgescu, \& Evans, 1991).

Large amounts of TNF- $\alpha$ are produced in the synovial membrane of afflicted joints where this cytokine promotes rapid proliferation of synoviocytes (synovial hyperplasia) and subsequent invasion into other tissues and the joint space causing swelling and pain (Harney et al., 2003). In afflicted joints, the proliferating synovium forms a tissue structure called a pannus and the synoviocytes are perpetually active and produce growth factors and matrix degrading enzymes, such as matrix-metalloproteinases (MMPs) that promote bone and cartilage erosion (Bucala, 2011; Mix, Sporn, Brinckerhoff, Eyre, \& Schurman, 2004). MMPs are expressed in response to inflammatory signals in the synovium and are responsible for degrading cartilage (Frisenda et al., 2013; Iwamoto, Okamoto, Toyama, \& Momohara, 2008). TNF- $\alpha$ not only plays a regulatory role for cytokine and MMP 
production in immune and resident cells in RA, but it also serves as a mediator for production of various chemokines that serve to attract more immune cells to the affected area. Chemokine producing cells respond robustly to inflammatory signals and produce chemokines that help perpetuate and expand the chronic inflammatory immune response (Iwamoto et al., 2008). Inhibition of TNF- $\alpha$ has been shown to reduce cellular invasion and hyperplasia in affected tissue by downregulating the inflammatory response (O'Kane et al., 2008). This is the basis for much of the current treatment methods for RA.

TNF antagonists can significantly slow the progression of multiple rheumatic and inflammatory diseases and improve quality of life. However, they are expensive and can results in dangerous side effects. The worst of these side effects include drug induced lupus and immune repression. TNF is utilized throughout the body as an important anti-tumor and anti-viral signal (Firth \& Critchley, 2011; Ozer \& Ozbalkan 2010). New and better treatments must be sought for all these reasons, and utilizing transcription factors may be the next important step in RA treatment.

Targeting transcription factors (small proteins that modulate gene expression) regulating production of the cytokines and other genes involved in induction of RA may provide more specific drug targets. Nuclear Receptor 4A2 (NR4A2) has arisen as a potential target of new treatments for RA. This transcription factor belongs to the nuclear receptor (NR) super-family; it has many functions in the body, one of which is modulation of inflammatory signaling via cytokines such as IL-8 and TNF (Aherne et al., 2009; Davies et al., 2005; Wang \& Wan, 2008). NR4A2 has also been found to be expressed at elevated levels in arthritic tissues removed from patients undergoing joint replacements (Mix et al, 2004). In addition to mediating inflammatory signaling pathways, NR4A2 has also been shown to respond to the DMARDs used for RA, psoriasis and similar diseases. Most notably, methotrexate has been shown to modulate NR4A2 levels suggesting that the disease alleviation provided by the drug may actually work through NR4A2 (McMorrow \& Murphy, 2011).

NR4A2 is one of the early rising proteins in response to inflammation; it has been shown to respond rapidly in vitro to inflammatory mediators when tested in synoviocytes and chondrocytes. NR4A2's transcriptional regulatory activity upregulates angiogenesis promoting factors and joint degradation factors: IL-8 and MMP-13 in synoviocytes (Davies et al., 2005). However, in chondrocytes, NR4A2 seems to serve an opposing function whereby it suppresses cartilage degrading factors MMP-1,-3, and -9 (Mix et al., 2007). Because of these different activities in vitro, it is important to investigate NR4A2's functions in an in vivo model of arthritis.

Mice serve as a suitable model for RA because the disease progression in mice mimics the progression in humans fairly closely; disease starts with inflammation and swelling caused by synoviocyte hyperplasia followed by cartilage destruction until the end stages of disease where most human patients seek surgical solutions (Asquith et al., 2009). Also, NR4A2 is conserved in mice, so it will be possible to evaluate the role of this gene in the disease process. In the current study, we established an expression profile of NR4A2 in two different mouse models of RA, the antigen induced arthritis model (AIA) and the serum transfer arthritis model (STA). We obtained joint tissues from mice at different stages of disease and examined NR4A2 protein levels and distributions in cartilage and synovial tissues by immunohistochemistry. We used two models that function through distinct mechanisms in order to have internal experimental comparisons for the role of NR4A2. The two models differ from one another in that AIA is induced by exposure to a foreign antigen known to elicit the RA autoimmunity response, and STA is induced by injecting experimental mice with serum from a genetically engineered strain of mice that spontaneously develop RA.

This study is the first to examine NR4A2 protein expression throughout the course of disease in two different mouse models of RA. We hypothesized that NR4A2 may be expressed early in chondrocytes, possibly working to protect the cartilage. In contrast, at later points in disease, NR4A2 levels may decrease in chondrocytes, potentially leading to cartilage destruction. We also hypothesized that the transcriptional activity in synoviocytes may function differently. As NR4A2 expression increases in the synovium, we hypothesized that joint destruction would continue along with the up-regulated expression since NR4A2 increases expression of inflammatory and cartilage degrading cytokines and proteinases. After peak expression, hypothesized to be early in chondrocytes and at mid stage in synoviocytes, we expected the levels of NR4A2 to decrease as the disease progressed to end stage.

To test our hypothesis, we established a method to quantify NR4A2 protein expression in these models. We used immunohistochemical staining with antibodies specific for NR4A2 which produce a brown stain on any cell expressing NR4A2. Expression levels were quantified in cartilage and synovial tissue from mice at different stages of disease. Initially, manual scoring was conducted on high resolution images to obtain an estimate of NR4A2 expression for each sample. The sample images were later subjected to automated scoring using ImagePro software. Our results indicate that NR4A2 levels were constant during early and mid stages of AIA, followed by a significant decrease in expression at late stages in both cartilage and synovium. In contrast, NR4A2 expression was not detected at any stage in the STA model. Manual and automated scoring methods produced similar results, however the error associated with manual scoring was greater.

\section{Results}

\section{Manual Scoring Results of the AIA model}

The image analysis of 14 AIA slides revealed NR4A2 protein expression to be highest in both synovium and cartilage through early and mid time points with no significant difference between the two. Synovium NR4A2 expression reached a peak of approximately $50 \%$ positive (at day 10), and the cartilage reached a peak of approximately $70 \%$ positive (at day 8 ). There was a significant decrease $(\mathrm{p}<0.001)$ in NR4A2 expression levels at the late time periods in both tissues. In comparison to the experimental samples, IgG control samples were all negative. Figure 1 below displays a comparison of one NR4A2 positive sample and one IgG control both with manual scoring templates overlaid. This template was used as an initial method of scoring images for 
NR4A2 expression. This method is discussed in full in the experimental procedures, and figure 2 contains the final NR4A2 scoring results from the manual counting method.

A

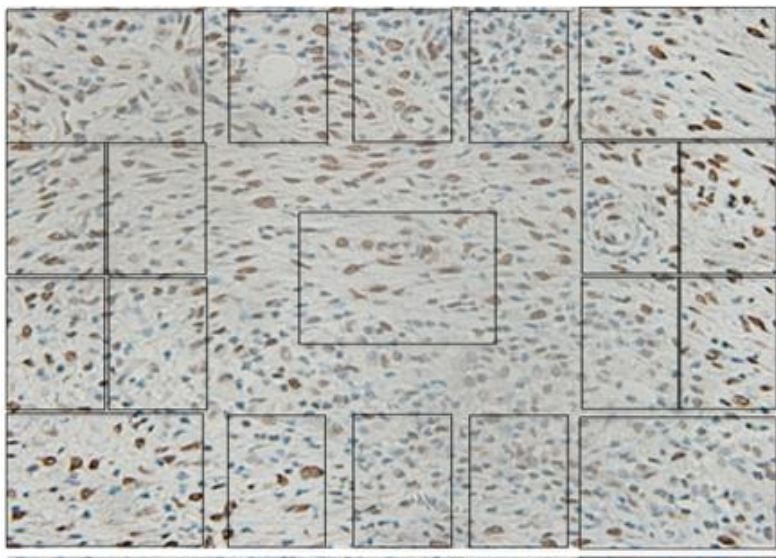

B

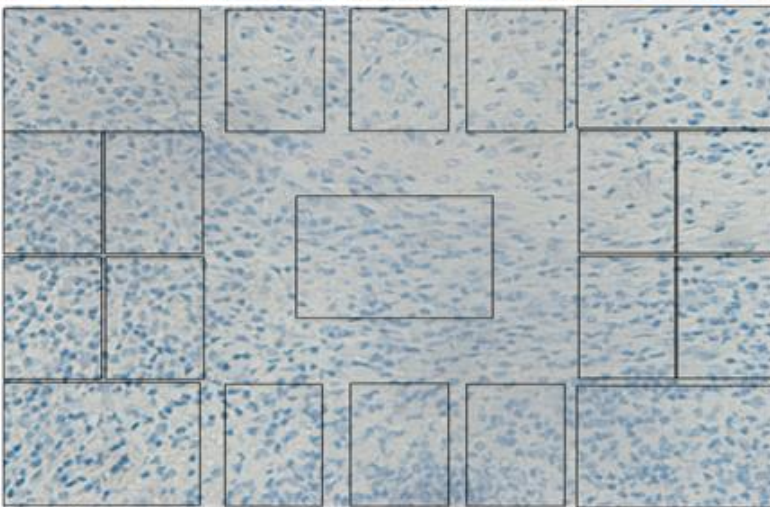

Figure 1: Method for quantifying NR4A2 expression: The images above are examples of the final manual scoring method and a comparison between the experimental stain and the $\mathrm{IgG}$ control stain. Image $\mathrm{A}$ is the experimental joint slice, and image B is a control joint slice. Though these particular images did not warrant use of the half boxes, some of the images required extensive use of the half boxes to gather enough data. These images are of the day 8 sample, which is early in disease progression. All the scores resulted in approximately $50 \%$ + for NR4A2 (+/- approximately $14 \%$ ).
A

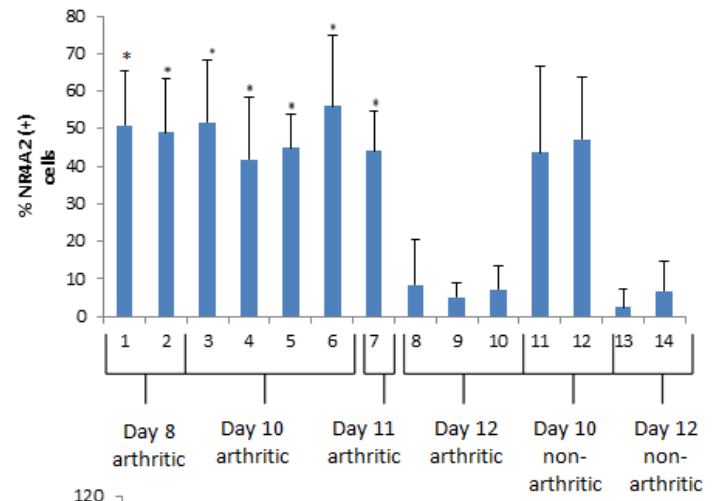

B

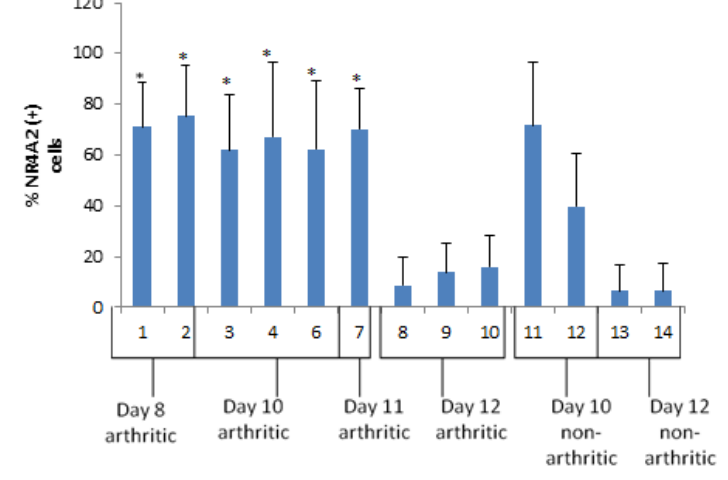

Figure 2: NR4A2 quantification results from synovium in the AIA and STA models: The graphs above present the combined manual scoring data for the synovium (A) and cartilage (B) NR4A2 expression. Quantification scores from 6 scorers are averaged together, and then are grouped by time-point of disease. Error bars are the standard deviations from the scores for each slide. The results are given as percentage of cells in the image expressing NR4A2. The t-test was run on early and mid manual scores in comparison to the late stage arthritic and non-arthritic control only. $* \mathrm{p}<0.001$. It should be noted again that the cartilage of slide 5 was too degraded to quantify, so for graph B, n=3 for day 10 arthritic instead of 4 as indicated in table 1.

There was also a significant difference between the mid and early time point expression levels and the late stage controls $(p<0.001)$. The late stage arthritic and non-arthritic samples both expressed less than 10\% NR4A2 in both synovium and cartilage. However, the mid stage controls expressed NR4A2 between $20-35 \%$ in synovium and $35-45 \%$ in cartilage. Figure 3 displays representative images of early stage and late stage synovium and cartilage samples from the AIA model. 

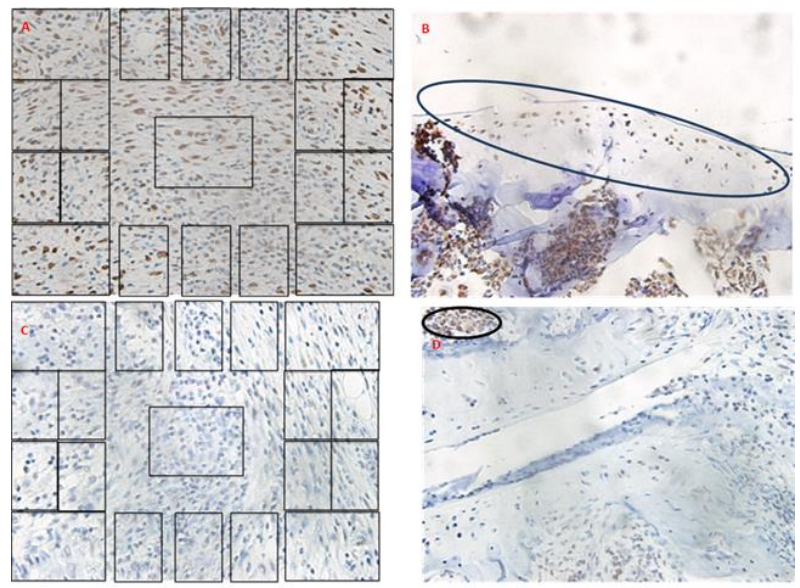

Figure 3: Day 8 and 12 arthritic synovium and cartilage: Image $A$ is a $400 x$ view of day 8 synovium. The brown stain indicates NR4A2 presence, and it is highly expressed in this image: approximately 53\% NR4A2+ (+/- approximately $17 \%$ ). Image $\mathrm{B}$ is a $400 \mathrm{x}$ picture of the left side of the articular cartilage section of the same joint at the femur head. There should be a complementary surface on the top of the image which would be patellar cartilage, but this sample suffered from significant tissue degradation. The circled area is the cartilage surface that was quantified. Image $C$ is a $400 x$ picture of day 12 synovium. There is no brown stain indicating no NR4A2 expression. Image D is a $400 \mathrm{x}$ picture of the right side of the articular cartilage section of the same joint at the femur head and patella. The complementary patellar was not degraded in this image as it was in the day 8 cartilage image. Again, a lack of brown stain indicates no expression of NR4A2. The exception is the brown staining in the marrow of the bone, circled at the top left. This was not part of our analysis, so the score for this image is still $0 \%$ NR4A2 expression.

\section{Automated Scoring Results from the AIA model}

The NR4A2 quantification scores established using ImagePro confirmed what our manual scoring suggested: that NR4A2 protein expression is highest in both synovium and cartilage through early and mid time points with no significant difference between the two. Synovium NR4A2 expression reached a peak of approximately $70 \%$ positive (at day 8 ), and the cartilage reached a peak of approximately $63 \%$ positive (at day 10). Though these results are different from the manual scoring data, the trends of both synovium and cartilage results held up. The NR4A2 expression was highest across early and mid time-points in disease with no significant difference between the two. Further, the automated results remained within the error from the manual scores with only one exception. The significant decrease in NR4A2 expression levels at the late time periods in both tissues also held up in the automated scoring. See figure 4 below for full details of the automated scoring results in comparison with the manual scoring.

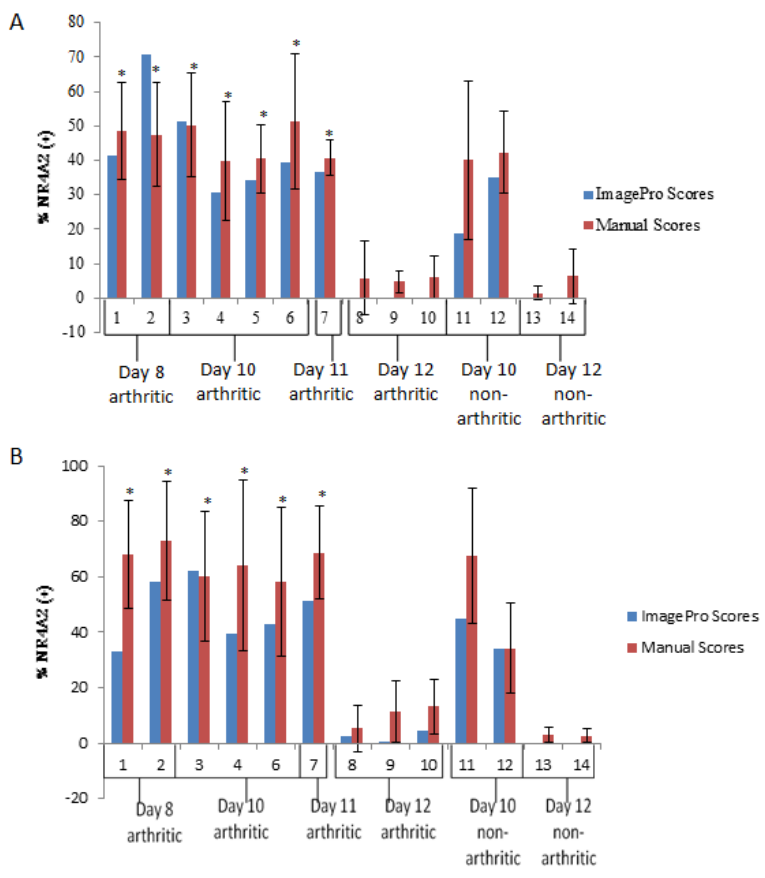

Figure 4: Synovium and cartilage score method comparisons from the AIA model: The graphs above are representations of the direct comparison between the ImagePro analysis and the manual scoring of each image of the synovium (A) and cartilage (B) of the sample joints. ImagePro analysis yielded consistently lower results than manual scoring, but within error for most of the samples. The t-test was run on manual scores only since there are not enough samples for meaningful statistics on the ImagePro analyses

\section{Results from the STA model}

In contrast to the AIA model, NR4A2 was not detected at any point in the STA model; figure 5 displays low and high power magnifications of STA method tissue samples. There is evidence of arthritic progression: joint space narrowing, swelling, pannus formation, and synovial invasion, but there is no NR4A2 protein expression. 


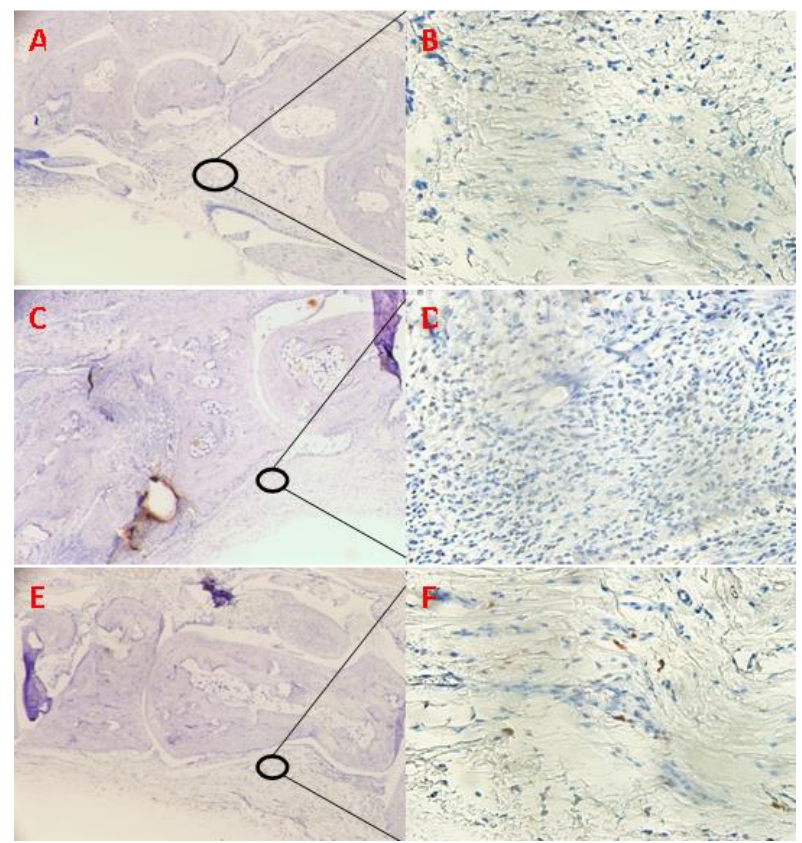

Figure 5: Representative images of activity from three joints in the STA model: Figures A, C, and E are low power images (dissecting scope: $6.3 \mathrm{x}$ ), and figures $\mathrm{B}, \mathrm{D}$, and $\mathrm{F}$ are high power (light microscope: 400x) enlargements of the images on the left. Images A and B are day 5 arthritic samples with a clinical score of 8 . Figures $\mathrm{C}$ and $\mathrm{D}$ are day 18 arthritic samples with a clinical score of 8 as well. Figures $\mathrm{E}$ and $\mathrm{F}$ are of the non-arthritic control at day 21 with a clinical score of 0 . There is bone deformation and pannus formation, but NR4A2 protein expression is absent in all of the samples from the STA model.

\section{Discussion and Future Goals}

NR4A2 expression in the AIA model was consistent with our hypothesis. We expected NR4A2 levels to be the highest at the early time points in cartilage and decline as the disease progressed, and we expected the levels in synovium to be the highest in the middle of disease progression. We found that there is no significant difference between the early and middle time points in either tissue, but there is a significant drop in NR4A2 expression at late stage in disease in both tissues which supports our hypothesis. Between the two tissue types, there is approximately a $20 \%$ difference at peak levels. There is not a significant difference between early and mid time points in cartilage, but there is a slight decline. Perhaps with more samples, a better defined trend might be revealed. If this decline in N4A2 is deemed significant in future studies, it also would be consistent with our hypothesis which predicted decreased NR4A2 levels in chondrocytes as disease progressed, allowing for degradation of cartilage.

Though we have shown trends in NR4A2 expression in the AIA model of RA, and others have also shown NR4A2 expression in RA, there is no denying that this is still a small study. More experimentation is needed to truly explain the role NR4A2 in RA. One of our main issues in this study was analyzing the data without enough samples to perform statistics on the ImagePro analyses. The student's T test was performed on the manual scores since there were six replicates for those data sets. Even with the wide error among those data, there was still a significant decrease in NR4A2 in the end stages of disease. This could indicate that the inflammatory response is diminishing as the end stages of disease set in when the cartilage has been mostly degraded, and the bones are grinding together or fusing, immobilizing the joint.

One limitation of this study was the unexpectedly high levels of NR4A2 in the day 10 control mice. Since there were only two day 10 controls it is difficult to interpret these results. It is possible that the slides were mislabeled or there was an error during the IHC procedure. A greater number of non-arthritic controls will be included in future studies to clarify these results.

Though both AIA and STA models showed clear progression of arthritis, only the AIA model arthritic mice expressed NR4A2. This is a puzzling result since NR4A2 has been shown to be expressed in the STA model of RA by other researchers, and NR4A2 expression in their experiments was affected by known RA attenuating treatments (such as methotrexate) suggesting it does indeed serve a regulatory role for RA in both animal models supporting its hypothesized role in human RA (Ryan et al., 2013). The important question is: why did the STA samples not show expression of NR4A2? They were stained on the same day, alongside the AIA samples. If that model stained successfully, the STA should have stained successfully as well. The most plausible explanation is that this model does not stimulate the same effects in the same cell types. Ryan et al. examined inflammatory gene responses including NR4A2 in monocyte and chondrosarcoma cell lines, not normal chondrocytes or synoviocytes. Further background research and experimentation with this model will be necessary to answer all of our questions concerning the STA model.

The analysis of the manual scores compared to the ImagePro scores revealed a consistently higher positive score from the manual score set. This is most likely to due to grey or dark blue stained cells appearing grey. Judging a whole group of cells on an image with the naked eye is incredibly subjective. Upon analysis in ImagePro, many of the cells that appeared dark brown were actually a dark grey or dark blue which could be discerned on the close up view mentioned in the methods. These colors can seem very much alike and caused many situations where a judgment call was necessary on the part of the manual scorers. Much of the wide error among the manual scores was from differences in these types of calls. Another source of manual scoring error was including objects in the count that may or may not have been cells. During group discussion there was often some disagreement about exactly what constituted a cell. It seemed that some of the students more than others included more fragments or other small anomalies on the image as cells in their whole cell counts. The scores for each image varied in both positive cells and total cells which then skewed percent positive results from the students. Based on what we have seen from the manual scores and the comparison to ImagePro, all future image analysis will be conducted with ImagePro because it is both more efficient and more reliable.

Developing an active animal model will allow us to answer more specific research questions. We know that NR4A2 is present in the arthritic joint at elevated levels and 
that its expression is modulated by anti-rheumatic drugs. The primary question is: how does NR4A2 directly affect RA? To answer this question we plan to develop transgenic mouse models with elevated and knocked out NR4A2 to examine how RA develops under these conditions. We expect RA conditions to be exacerbated by increased NR4A2 due to its inflammatory activity regulation, and we expect RA damage will be lessened in mice with knocked-out NR4A2 due to the lack of inflammatory signaling.

\section{Experimental Procedures}

\section{Tissues}

All tissue sections examined were provided by Dr. Ellen Gravallese (University of Massachusetts Medical School, Department of Rheumatology) from ongoing studies with the murine AIA and STA models. Tissues were decalcified, formalin-fixed and imbedded in paraffin. Sections were cut at a thickness 20 microns, mounted on glass slides, and stored at 4C. Tissues used in this study were approximately 1-1.5 years old. Three small sets of preliminary slides $(2$ or 3 samples from each model) stained for IHC were examined in order to establish protocols for NR4A2 IHC, imaging and quantification. Each set contains mouse cross sections from knees (AIA) and ankles (STA) of the mice. Once the protocols were established, two larger sets of slides with mice at early mid and late stage were obtained. Time-points for the AIA model are day 8 (early), day 10/11 (mid) and day 12 (late); non-arthritic controls were sacrificed at days 10 and 12 alongside experimental samples. The time-points for the STA model are day 5 (Early), day 7/8 (mid) and day 18 (late); the non-arthritic control in this model was taken at day 21 . These times are well established for each of these models, and more slide information can be found below in tables 1 and 2 (Asquith et al., 2009; Brackertz, Mitchell \& Mackay, 1977).

Table 1 is a list of the slides from the AIA model, their experimental conditions, and clinical scores. Clinical scores are noted as peak knee swelling as the disease progressed in the individual mouse (it is not necessarily the same as the takedown day).

Table 2 is a list of the slides from the STA model, their experimental conditions, and clinical scores. Clinical scores are assigned based on swelling and degradation of each limb of the mouse and combined total score out of 12 possible points ( 4 per limb). Slides 3 and 4 are serial sections from the same mouse as are 5 and 6 from a separate mouse and slides 6-12 as well.

\section{Immunohistochemistry (IHC)}

All IHC staining was conducted by Helena PappasLeBeau at the Tulane University School of Medicine Histology Core Laboratory. All slides were heated overnight at $60 \mathrm{C}$ to adhere tissues prior to staining. Slides were quenched in $3 \% \mathrm{H}_{2} \mathrm{O}_{2}$ and methanol for $5 \mathrm{~min}$ and rinsed in $\mathrm{dIH}_{2} 0$. Antigen retrieval was conducted in target retrieval solution (Reveal, BioCare) heated to $95 \mathrm{C}$ for $35 \mathrm{~min}$. Slides were cooled for $20 \mathrm{~min}$ and rinsed in $\mathrm{dIH}_{2} \mathrm{O}$. Slides were processed for immunohistochemistry with the BioCare Nemesis 7200 autostainer. Briefly, slides were blocked $\left(\mathrm{H}_{2} \mathrm{O}_{2}\right.$, 5 min; Avidin, $10 \mathrm{~min}$; Biotin, $10 \mathrm{~min}$, Sniper protein block (BioCare), $10 \mathrm{~min}$ ), followed by incubation with primary rabbit polyclonal IgG antibodies for $60 \mathrm{~min}$ (Santa-
Cruz Biotechnology, NR4A2 N-20 antibody (sc-991 or normal rabbit IgG (sc-2027). Negative control slides were also processed with an $\operatorname{IgG}$ control antibody at the same concentration. The secondary antibody was incubated with slides for $30 \mathrm{~min}$ (Rabbit-on-rodent HRP-Polymer, \#902RMR622-111210, Biocare Medical). The beta-DAB substrate was applied to cells for 2 minutes. Slides were counterstained with CAT Hematoxylin (Biocare Medical, \#CATHE-6L) followed by Tacha's Bluing Solution (Biocare Medical, \#HTBLU-MX). Slides were dried, treated with xylene, and mounting media and cover slips were applied. Stained slides were stored at room temperature. This procedure renders all cells blue, but the NR4A2 positive cells stain brown over the blue if the primary antibody adhered to the target molecule in the cell. The HRP compound that is attached to the secondary antibody produces the brown stain when it interacts with the beta-DAB substrate.

Table 1: Clinical data from AIA model

\begin{tabular}{lccc}
\hline Slide & $\begin{array}{c}\text { Disease } \\
\text { Condition }\end{array}$ & $\begin{array}{c}\text { Takedown } \\
\text { day }\end{array}$ & $\begin{array}{c}\text { Peak Knee } \\
\text { swelling (mm) }\end{array}$ \\
\hline 1 & Arthritic & Day 8 & 1.28 \\
\hline 2 & Arthritic & Day 8 & 1.11 \\
\hline 3 & Arthritic & Day 10 & 0.37 \\
\hline 5 & Arthritic & Day 10 & 0.56 \\
\hline 6 & Arthritic & Day 10 & 0.56 \\
\hline 7 & Arthritic & Day 10 & 0.56 \\
\hline 8 & Arthritic & Day 11 & 1.22 \\
\hline 9 & Arthritic & Day 12 & 0.73 \\
\hline 10 & Arthritic & Day 12 & 0.46 \\
\hline 11 & Arthritic & Day 12 & 0.73 \\
\hline 12 & Non-arthritic & Day 10 & n/a \\
\hline 13 & Non-arthritic & Day 10 & n/a \\
\hline 14 & Non-arthritic & Day 12 & n/a \\
\hline
\end{tabular}

Table 2: Clinical data from STA model

\begin{tabular}{llll}
\hline Slide & $\begin{array}{l}\text { Disease } \\
\text { Condition }\end{array}$ & $\begin{array}{l}\text { Takedown } \\
\text { day }\end{array}$ & Clinical score \\
\hline 1 & Arthritic & 5 & 8 \\
\hline 2 & Arthritic & 5 & 8 \\
\hline 3 & Arthritic & 7 & 10 \\
\hline 4 & Arthritic & 7 & 8.5 \\
\hline 5 & Arthritic & 8 & 8.5 \\
\hline 6 & Arthritic & 8 & 9 \\
\hline 7 & Arthritic & 18 & 9 \\
\hline 8 & Arthritic & 18 & 9 \\
\hline 9 & Arthritic & 18 & 9 \\
\hline 10 & Arthritic & 18 & 9 \\
\hline 11 & Arthritic & 18 & 9 \\
\hline 12 & Arthritic & 18 & 9 \\
\hline 13 & Arthritic & 18 & 8 \\
\hline 14 & Arthritic & 18 & 8 \\
\hline 15 & Non-arthritic & 21 & 0 \\
\hline
\end{tabular}




\section{Imaging procedure}

The imaging program CellSens Standard was used in conjunction with an Olympus BX51 microscope and Olympus DP72 digital camera mounted to the scope. Access to the microscope/camera unit and the computer with CellSens was provided by Dr. Rosalie Anderson. Most of the settings remained the same throughout the imaging process: exposure settings: Full region, ISO at 800 , and 0 compensation; Resolution setting at 4140x3096. Contrast settings in CellSens were adjusted on a subjective basis; the image from some slides was clearer with the contrast setting on vivid, and some images were clearer with contrast set on natural. The images were captured from a range from $100 x$ to $400 x$ magnification and saved as JPEG files.

\section{Manual quantification method}

The 400x images of the synovium and cartilage interface at the patella were printed in color on 8.5"x11" glossy paper at the Loyola University print shop. Prints were from files placed in PowerPoint and dragged to 6" 8 " (on the scale bars in PowerPoint) in a template. In order to get an accurate estimated cell count estimate, five equal sections were drawn onto the prints of the images. Using the resolution of the image and the pixel density, which were retrieved from the properties menu on each image, and the formula, [(length in pixel) $\mathrm{x}(1000 \mu \mathrm{m} / \mathrm{cm})] /$ [(resolution in pixels $/ \mathrm{cm}) \mathrm{x}$ (magnification)], the actual dimensions represented in the picture were calculated. The total area was approximately $9.97 \times 10^{4} \mu \mathrm{m}^{2}$, and the area of each box was approximately $6229.5 \mu \mathrm{m}^{2}$. All cells within the sections were counted and the cells stained brown indicating positive presence of NR4A2 were counted. The sections were averaged together to obtain an average percent positive for the image.

In the AIA model the areas of interest in the knee were the synovium (S), the femur/tibia area (FT) and the patella/femur head area (PF). Due to issues with the tissue quality only the $\mathrm{S}$ and $\mathrm{PF}$ areas were quantified. When examining the PF area it became necessary to split the area into multiple panel images ( 3 or 4 depending on the width of the joint) to achieve a full view of the area: the femur head and the patella body. Since the joint curves, many of the images overlapped in sections and had to be edited so that the overlapped areas were only counted in one of the panels. These PF images are not counted with estimation boxes; instead, they are directly counted all the way across the surface of the joint because there are fewer cells in the PF joint.

Some of the tissue suffered quality issues, so the quantification had to be altered. Many of the slides' synovium were degraded and did not give a full field of cells to quantify. Therefore, half estimation boxes were added to the quantification template; refer to figure 1 in the results for an example of the final modified, manual scoring template. These were to be used if the five main boxes did not lie over a full field of cells. If one of the main boxes was approximately half empty, the nearest half box would be used to fill in as a substitute; if a whole main box was empty, the two nearest useable half boxes would be used to substitute. The goal was to have five boxes' worth of data for each synovium section. The cartilage sections were imaged as panels again and counted directly just as the first full set.
Six student volunteers counted all of the images collected using this manual scoring method. The averages of all the manual scores were graphed, and Student's t-test was performed to test for significant differences between controls and different arthritic time-points. After reviewing the results, two of the volunteers' scores were excluded as outliers, and the data sets were re-examined with their data excluded.

Once we finally established a functional procedure for digitally analyzing images using ImagePro, all the images from the two full sets and compared those scores to the original estimations. The area for all five boxes is $3.12 \times 10^{4}$ $\mu \mathrm{m}^{2}$, and $\left(9.97 \times 10^{4} \mu \mathrm{m}^{2} / 3.12 \times 10^{4} \mu \mathrm{m}^{2}\right)$ equals approximately 3.199. The estimated scores were multiplied by the factor of 3.199 in order to compare total cell and positive cell counts to the digital scores. The scores were compared directly to manual scores of the images.

\section{Automated scoring}

Automated NR4A2 expression scores were obtained using ImagePro, an image analysis and capture software from Media Cybernetics. Two protocols were used for ImagePro scoring. First a whole cell count was obtained by separating the image into the RGB color channels, and the count was performed on the individual channel image with the most contrast between the cells and the background: most often the Red channel. This count was done with a size limitation given in area of pixels. The lower limit was established to be effective set at 400 square pixels, and the upper limit was established at 40,000 square pixels.

Once the whole cell count was obtained, a count of positive cells was obtained on the original file still in color with the eye-dropper tool. This was used to select any brown positive indicator and have the program select and highlight any cell expressing that defined pixel range. A preview box in the selection menu allows the user to scan an area of $3 \times 3$ pixels in order to determine what the true color is in a very finite group of pixels. Once the range of positive indicating color was selected, the count was performed and results were recorded.

\section{Acknowledgements}

First and foremost, I would like to thank my advisor Dr. Mix. She has worked with me for two years, guided me through the research process, and taught me more than I can express. I would also like to thank the rest of the Biology Department as a whole. This group of faculty and staff have treated me as one of their own and created a supportive, nurturing environment for budding scientists that has been instrumental in my success at Loyola. I must also acknowledge the never-ending support given by my parents and my significant other, Tiffany. They have never let me down. Additionally I would like to thank Kate Birdwhistell, Nicole Daniel, Jessica Richmond, Safa Raouf, Saba Raouf, and Megan Stevens, the volunteers who scored all those joint images. Finally, a thank you is owed to the Tulane Pathology Core Lab for conducting the immunohistochemical staining and to Dr. Ellen Gravallese for providing us with our samples. Support for this project was provided by a grant from the Louisiana Board of Regents. 


\section{References}

Aherne, CM, McMorrow, J, Kane, D, FitzGerald, O, Mix, KS and Murphy, EP. (2009). Identification of NR4A2 as a transcriptional activator of IL-8 expression in human inflammatory arthritis. Molecular Immunology, 46(16), 3345-3357.

http://www.ncbi.nlm.nih.gov/pubmed/19732956

Asquith, DL, Miller, AM, McInnes, IB and Liew, FY. (2009). Autoimmune disease: Rheumatoid arthritis; Animal models of rheumatoid arthritis. European Journal of Immunology, 39(8, 1991-2058.

http://www.ncbi.nlm.nih.gov/pubmed/19672892

Brackertz D, Mitchell, GF, and Mackay, IR. (1977). Antigeninduced arthritis in mice.Arthritis and Rheumatism, 20(3), 841-50.

http://onlinelibrary.wiley.com/doi/10.1002/art.1780200314/ abstract

Bucala, R. (2011). Intersecting Roads to Rheumatoid Arthritis. Arthritis \& Rheumatism, 63(7), 1770-1772. http://www.ncbi.nlm.nih.gov/pubmed/21717419

Carr, AJ. (2003). The genetic basis of severe osteoarthritis. Annals of the Royal College of Surgeons of England, 85(4), 263-268.

http://www.pubmedcentral.nih.gov/articlerender.fcgi?artid= 1964403\&tool=pmcentrez\&rendertype $=$ abstract

Davies, MR, Harding, CJ, Raines, S, Tolley, K, Parker, AE, Downey-Jones, M, and Needham, MR. (2005). NURR1 dependent regulation of pro-inflammatory mediators in immortalized synovial fibroblasts. Journal of Inflammation, 2,15 .

http://www.pubmedcentral.nih.gov/articlerender.fcgi?artid= $1308852 \&$ tool $=$ pmcentrez\&rendertype $=$ abstract

Firth, J and Critchley, S. (2011). Treating to target in rheumatoid arthritis: biologic therapies. British Journal of Nursing, 20(20), 1284-1291.

http://www.ncbi.nlm.nih.gov/pubmed/22068002

Frisenda, S, Perricone, C, and Valesini, G. (2013). Cartilage as a target of autoimmunity: A thin layer. Autoimmunity Reviews, 12(5), 591-598.

http://www.ncbi.nlm.nih.gov/pubmed/23201917

Harney, SMJ, Newton, JL and Wordsworth, BP. (2003). Molecular genetics of rheumatoid arthritis. Current Opinion in Pharmacology, 2003. 3(3), 280-285. http://linkinghub.elsevier.com/retrieve/pii/S147148920300 $\underline{0493 .}$

Hulkower, KI, Georgescu, HI and Evans, CH. (1991). Evidence that responses of articular chondrocytes to interleukin-1 and basic fibroblast growth factor are not mediated by protein kinase C. The Biochemical Journal, 276(1), 157-162.

http://www.pubmedcentral.nih.gov/articlerender.fcgi?artid= $1151158 \&$ tool=pmcentrez\&rendertype $=$ abstract.

Iwama, H, Gojobori, T and Li, WH. (2004). Highly Conserved Upstream Sequences for Transcription Factor Genes and Implications for the Regulatory Network. Proceedings of the National Academy of Sciences, 101(49), 17156-17161.

http://www.jstor.org/stable/3373976?origin=JSTOR-pdf.

Iwamoto, T, Okamoto, H, Toyama, Y, and Momohara, S. (2008). Molecular aspects of rheumatoid arthritis: chemokines in the joints of patients. Federation of
European Biochemical Societies Journal, 275(18), 44484455. http://www.ncbi.nlm.nih.gov/pubmed/18662305

McMorrow, JP and Murphy, EP. (2011). Inflammation: a role for NR4A orphan nuclear receptors? Biochemical Society Transactions, 39(2), 688-693. http://www-06.allportland.net/bst/039/0688/0390688.pdf

Mix, KS, Sporn, MB, Brinckerhoff, CE, Eyre, D, and Schurman, DJ. (2004). Novel Inhibitors of Matrix Metalloproteinase Gene Expression as Potential Therapies for Arthritis. Clinical Orthopaedics and Related Research, 427, 129-137. http://www.ncbi.nlm.nih.gov/pubmed/15480055

Mix, KS, Attur, MG, Al-Mussawir, H, Abramson, SB, Brinckerhoff, CE, and Murphy, EP. (2007). Transcriptional repression of matrix metalloproteinase gene expression by the orphan nuclear receptor NURR1 in cartilage. Journal of Biological Chemistry, 282 (13), 9492-9504. http://www.ncbi.nlm.nih.gov/pubmed/17283078

O'Kane, M, Markham, T, McEvoy, AN, Fearon, U, Veale, DJ, FitzGerald, O, Kirby, B and Murphy, EP. (2008). Increased Expression of the Orphan Nuclear Receptor NURR1 in Psoriasis and Modulation following TNF- $\alpha$ Inhibition. Journal of Investigative Dermatology, 128(2), 300-310. http://www.ncbi.nlm.nih.gov/pubmed/17671512

Ozer, $\mathrm{H}$ and Ozbalkan, Z. (2010). Clinical Efficacy of TNF- $\alpha$ Inhibitors: An Update. International Journal of Clinical Rheumatology, 5(1), 101-115. http://www.medscape.com/viewarticle/717623

Ryan, SM, McMorrow, J, Umerska, A, Patel, HB, Kornerup, KN, Tajber, L...Brayden, DJ. (2013). An intra-articular salmon calcitonin-based nanocomplex reduces experimental inflammatory arthritis. Journal of Controlled Release, 167, 120-129.

http://www.ncbi.nlm.nih.gov/pubmed/23391443

Spector, TD and MacGreggor, AJ. (2044). Risk factors for osteoarthritis: genetics. Osteoarthritis and Cartilage, 12, 39-44.

http://www.sciencedirect.com/science/article/pii/S1063458 40300253X

Wang, K and Wan. Y. (2008). Nuclear receptors and inflammatory diseases. Journal of Experimental Biology, 233, 496-506. http://www.ff.ul.pt/FCT/PTDC/SAUFCF/102607/2008/14.pdf

No author listed. (2003). Diseases and Conditions: Rheumatoid Arthritis. Mayo Clinic. http://www.mayoclinic.org/diseasesconditions/rheumatoid-arthritis/basics/causes/con20014868

No author listed. (2011). News from the Arthritis Foundation. Arthritis Foundation: arthritis.org, http://www.arthritis.org/files/images/AF_Connect/Departm ents/Public_Relations/Arthritis-Prevalence-Fact-Sheet--37-12.pdf

No author listed. (2012). Drug Therapy for Rheumatoid Arthritis: Comparative Effectiveness. Agency for Healthcare Research and Quality,

http://effectivehealthcare.ahrq.gov/ehc/products/203/1315/rhe um arth clin fin to post.pdf

No author listed. (2013). Understanding RA Stages and Progression. RheumatoidArthritis.net, http://rheumatoidarthritis.net/what-is-ra/stages-andprogression/ 